\title{
The use of probiotics for improving lipid profiles in dyslipidemic individuals: an overview protocol
}

\author{
Patricia M. Fortes ${ }^{1,2}$, Solomar M. Marques ${ }^{2}$, Karolline A. Viana ${ }^{3}$, Luciane R. Costa ${ }^{3,4}$, Alessandra V. Naghettini ${ }^{2,5}$ \\ and Paulo Sucasas Costa ${ }^{1,2^{*}}$
}

\begin{abstract}
Background: Dyslipidemia is a major risk factor in triggering cardiovascular events, which can lead to the death of millions of people around the world. Thus, several pharmacological and non-pharmacological therapeutic strategies have been developed in recent decades with the objective of improving lipid profiles, including the use of probiotics. Therefore, the purpose of this protocol is to describe the steps that will guide the construction of an overview to demonstrate the scientific evidence of the efficacy of probiotics in improving the lipid profile of dyslipidemic individuals and to propose specific recommendations regarding their use.

Methods: The search will be conducted in the following databases: MEDLINE/PubMed, EMBASE, PROSPERO, Cochrane Library, CINAHL, JBI Database of Systematic Reviews and Implementation Reports, Google Scholar, and CADTH. Reviewers will select systematic evaluations and data analyses from randomized clinical trials that evaluated the effects of probiotics on lipid profiles. The studies will be analyzed for methodology quality by the AMSTAR 2 tool and risk of bias by ROBIS. The data will be extracted by three independent reviewers based on a data extraction sheet, which will include the most relevant variables for the analysis and interpretation of the results. The variables will be categorized and described in narrative form or in tables.

Discussion: There are some systematic reviews about the use of probiotics to prevent and/or treat dyslipidemia; however, their outcomes related to the ability of probiotics to improve lipid profiles are conflicting. So, an overview on this topic is needed to clarify this important issue.
\end{abstract}

Systematic review registration: PROSPERO CRD42017080328

Keywords: Probiotic, Dyslipidemia, Hypercholesterolemia, Overview, Umbrella review

\section{Background}

Dyslipidemia is one of the most important cardiovascular risk factors (CRF) in triggering serious events. According to the World Health Organization (WHO), 17 million people died in 2015 from cardiovascular disease, accounting for about $31 \%$ of deaths worldwide in that period; acute myocardial infarction (AMI) and stroke were the main events diagnosed as a cause of death [1].

\footnotetext{
* Correspondence: paulosucasas@ufg.br

${ }^{1}$ Health Sciences Graduate Program, Medicine School, Universidade Federal de Goiás (UFG), Rua 235, s/n, Setor Leste Universitário, Goiânia, GO CEP 74605-050, Brazil

${ }^{2}$ Department of Pediatrics, Hospital das Clinicas, UFG, Primeira Avenida, s/n, Setor Leste Universitário, Goiânia, GO CEP 74605-020, Brazil

Full list of author information is available at the end of the article
}

Therefore, dyslipidemia is still the most important modifiable risk factor associated with these events [2]. However, what makes controlling it challenging is that it has reached epidemic proportions, develops quietly, and is associated with other cardiovascular risk factors $[3,4]$.

Considering that the control of dyslipidemia is an important factor in the prevention of AMI and stroke, there are many protocols and guidelines with therapeutic regimens related to pharmacological and non-pharmacological treatments. Yet, controlling this condition has become increasingly difficult probably due to its multifactorial origin $[5,6]$. Therefore, new treatment options have been proposed; among them, some scientific interest has focused on the use of probiotics, defined as "living microorganisms that in

(C) The Author(s). 2018 Open Access This article is distributed under the terms of the Creative Commons Attribution 4.0 International License (http://creativecommons.org/licenses/by/4.0/), which permits unrestricted use, distribution, and 
adequate quantities are able to bring benefits to host's health" [7].

In recent decades, probiotics have been used in the management of different health conditions, demonstrating their benefits and safety when administered in humans, including dyslipidemic individuals [8]. The mechanisms of action by which different probiotic strains act in the control of dyslipidemia have been suggested from randomized clinical trials, highlighting (1) deconjugation and precipitation of bile acids by enzymatic action; (2) incorporation of cholesterol into the cell membrane of microorganisms; (3) inhibition of cholesterol transmembrane transporter expression in enterocytes; (4) cholesterol conversion to coprostanol; and (5) inhibition of hepatic synthesis of cholesterol [9].

Several studies, mainly clinical trials, have demonstrated the ability of some bacterial strains to reduce total cholesterol, low-density lipoprotein cholesterol (LDL-c), and triglycerides [10-15]. On the other hand, no changes were observed in the lipid profile after the use of probiotics in other studies [16-18]. In a preliminary literature search, we found systematic reviews (SRs) that generated contradictory results of probiotic use in dyslipidemic individuals [19-22]. Thus, an overview of SRs is required to support the healthcare decision-making on probiotics to treat dyslipidemia,

Therefore, the purpose of this protocol is to critically assess the scientific evidence presented in the systematic reviews currently available regarding the efficacy of probiotics in improving the lipid profile in dyslipidemic individuals. After this process, an overview will be drawn up showing the quality of such evidence that can enable health professionals to make specific recommendations for the use of probiotics in this context.

\section{Methods/design}

The term "overview" refers to a review of systematic reviews and/or meta-analyses that compares studies and promotes a critical analysis of the main viable evidence about a particular issue, thus facilitating appropriate decision making [23]. The development of this protocol followed the guidelines presented by the Joanna Briggs Institute (JBI) for reviewers [23] and the recommendations proposed by PRISMA-P (Preferential Reporting Items for Systematic Review and Meta-analysis-Protocol) [24] (Additional file 1). The elaboration of the overview will follow the steps described in this protocol, and any deviation in the steps proposed here will be reported and justified. This overview protocol was registered in the PROSPERO database CRD42017080328.

\section{Research question}

Does the use of probiotics improve the lipid profile in dyslipidemic individuals?

\section{Eligibility criteria}

Types of study

Only systematic reviews which have been carried out from randomized clinical trials with or without meta-analyses, performed or not under Cochrane methodology will be included to allow a greater level of evidence [25]. Additionally, systematic reviews may have clearly demonstrated a research question that includes the acronym PICO (population, intervention, comparison/control, outcome(s)) [23, 26]. The SRs should have been published since January 2000, because this is the period of the publication of one of the first SR summarizing evidence about efficacy of probiotic use in the control of dyslipidemia [27]. Narrative reviews, protocols, reviews of animal studies, reviews of observational studies, and case series will not be included.

\section{Participants}

The participants included in SR should be well described regarding characteristics such as age, gender, sample size, and clinical conditions associated with dyslipidemia. Systematic reviews will be excluded if more than $80 \%$ of the participants in the sample are carriers of type 1 diabetes, hypothyroidism, nephrotic syndrome, or if they are pregnant women or alcoholics, because they might require other therapeutic interventions that would confound the probiotics effect.

\section{Intervention}

The probiotic should be the only intervention used to control dyslipidemia. Systematic reviews will be included if they are detailed in the study design: administration of treatment, presentation form, probiotic strain identification, dose and time of probiotic use, whether the groups were conducted in parallel or cross-over, and if there were associations with other factors that could interfere with the action of the probiotic (e.g., prebiotic, symbiotic, phytotherapeutic, neutracetic, soy, drugs).

\section{Comparator}

The selected SRs should demonstrate that the interventions were compared with a control group composed of placebo or non-therapeutic substance.

\section{Outcomes}

We will include SRs whose outcomes were related to changes in lipid profiles (total cholesterol, LDL cholesterol, HDL cholesterol, and triglycerides).

\section{Search strategy}

Electronic databases

Two investigators (PMF and PSC) will carry out the searches using the following databases: PubMedMEDLINE (www.ncbi.nlm.nih.gov/pubmed/), EMBASE 
(www.embase.com), Cochrane Library (www.cochranelibrary.com), PROSPERO (www.crd.york.ac.uk), CINAHL, and JBI Database of Systematic Reviews and Implementation Reports. All electronic search tools are available for free, with the exception of EMBASE. Databases for gray literature such as Google Scholar (www.scholar.google. com) and CADTH "Gray Matters Light" (http:// www.cadth.ca//resources/finding-evidence/grey-matters) will be searched for non-indexed publications and government policies. The references cited in the selected studies will also be reviewed by manual search.

\section{Search terms}

For the overview, authors used the key elements of the research question to define the descriptors selection. The purpose of this selection is to define the keywords that best described the eligibility criteria of the SRs to be selected without significantly narrowing the search. After identification, the terms will be used in combination, including keywords (MeSH-Medical Subject Headings) and entry terms that are present in the title, abstract, and full text (Additional file 2). The combinations of keywords and search strategy are exemplified in Fig. 1 and will be repeated for all databases.

\section{Selection of studies}

Two independent reviewers (PMF and PSC) will select the studies. In case of disagreement between reviewers, a third reviewer will be consulted. Evaluation will begin with the exclusion of duplicate articles using the program Mendeley-Reference Management Software and Researcher Network, followed by application of the eligibility criteria (initially, the title and abstract). Selected articles from screening will undergo thorough reading for further examination of the eligibility criteria; eligible studies will be submitted for data extraction and quality assessment.

For a better visualization of the stages of study selection, a flow diagram will be set up as suggested by
PRISMA (Preferential Reporting Items for Systematic Reviews and Meta-analyses) [28].

\section{Extraction of data}

Each of the systematic reviews will be submitted to data extraction by three separate reviewers (PMF, SMM, and $\mathrm{KAV}$ ), thus ensuring that important data will not be lost. The reviewers will use a standard form that will be initially tested in three studies; if necessary, it will be adapted. In order to adequately meet the interests research question of this overview, the following data will be extracted: (1) author, country, year of publication, number of RCTs included, funding (if any), language, and database (including gray literature); (2) characteristics of the population (sex, age, baseline diseases) and sample size; (3) intervention-strains of probiotics, study design, duration of treatment, dose, presentation form, association (prebiotic, symbiotic); (4) comparators (placebo or yogurt); (5) outcomes-changes in serum levels of total cholesterol, LDL-cholesterol, HDL-cholesterol, and triglycerides; (6) main adverse events related to probiotic supplementation (e.g., systemic-bacteremia, sepsis; and gastrointestinal-diarrhea, flatulence, abdominal pain); and (7) results-qualitative and quantitative (means, standard deviations, effect size, confidence interval, $p$ value, and meta-analysis, if the data allow). If there are questions and disagreements between the reviewers in this process, a fourth reviewer will be consulted. If necessary, the RCTs analyzed in SRs will be checked for completion of some important data.

Assessment of the risk of bias and methodological quality The risk of bias will be evaluated using the ROBIS tool, according to specific guidelines for systematic reviews related to interventions. This tool is composed of three phases: phase 1-assessment of relevance (optional); phase 2-identification of concerns with the review process regarding the presence of bias, which is performed by four different domains and phase

Example: PUBMED / MEDLINE - 115 studies (search 29 Sept 2017).

"((probiotic).ti,ab OR (probiotics).ti,ab OR (probiotic agent).ti,ab OR (ferment milk).ti,ab OR (yogurt).ti,ab OR (yoghurt).ti,ab OR (Lactobacillus).ti,ab OR (Bifidobacterium).ti,ab) AND ((dyslipidemia).ti,ab OR (hypercholesterolemia).ti,ab OR (hyperlipidemia).ti,ab OR (low density lipoprotein cholesterol).ti,ab OR (dyslipoproteinemia).ti,ab OR (high cholesterol levels).ti,ab OR (elevated cholesterol).ti,ab OR (lipid profile).ti,ab)) AND ((systematic review).ti,ab OR (systematic review AND meta-analysis).ti,ab OR (meta-analysis).ti,ab)"

Fig. 1 Combination of keywords and entry terms for search in the database 
3-judgment of the risk of bias through the summary of phase 2 findings. With the exception of phase 1, the other phases present answers that vary in (Y) "yes," (PY) "probably yes," (PN) "probably not," (N) "no," and (NI) "not informed" contained in each sub-area of the domains. The reason for concern is the risk of bias classified as high, low, or unclear [29].

The tool AMSTAR 2 (Additional file 3) (https:// amstar.ca/docs/AMSTAR-2.pdf), which is composed of 16 domains, will be used in the evaluation of the methodological quality of the SRs. Of these 16 domains, 7 (2, $4,7,9,11,13$, and 15) are considered critical for the reliability of SRs results, ranking the SR in high, moderate, low, and critically low [30]. For each domain, the answers will vary in "yes" (all criteria required by the domain have been checked), "partially yes" (only part of the criteria required in the domain have been checked), and "no" (the criteria required by the domain were not checked). Multiple flaws in non-critical domains (more than 3) will be considered capable of decreasing confidence in results; in this case, the classification will start from the moderate level. The domains will be analyzed independently, so that the response to a particular domain does not influence the evaluation of the others [30].

Both the bias risk assessment and the methodological quality evaluation of the reviews will be carried out by two independent reviewers (PMF, KAV). If disagreements occur, a third reviewer will be consulted (SMM).

\section{Data synthesis}

Results of reviews will be summarized, and the characteristics of the variables will be described in narrative form or in tables where each one will be categorized and, if appropriate, allocated as a statistical measure. This will allow the comparison of studies, visualization of effects and critical interpretation of results.

A meta-analysis will not be performed, given that pooling the results of meta-analyses could introduce significant overlap and biased results, as a RCT could be included in more than one SR [31].

The calculation of the corrected covered area (CCA) will be applied to estimate the overlap of RCTs among the reviews included [31]. In order to calculate the CCA, a citation matrix containing the primary articles and the revisions will be elaborated and arranged in the order of publication of the revisions. If there is a high overlap of RCTs, the most recent revisions will be maintained and the impact of such findings on the evidence will be discussed.

The discordant results presented among SRs will be analyzed based on the algorithm proposed by Jadad et al. [32], which seeks to identify the likely causes of contradictory results in reviews that presented the same research question. Subsequently, it will be discussed how these different causes of disagreement between the results impact on the evidence.

\section{Discussion}

The increase in the number of dyslipidemic individuals due to changes in life habits in all age groups has become a challenge for the control of this important cardiovascular risk factor [1]. Consequently, more and more investigations are carried out seeking therapeutic alternatives that can prevent the onset of cardiovascular events. Therefore, the purpose of this protocol is to present the search pathway for a critical analysis of current scientific evidence about the efficacy of probiotics use in the control of dyslipidemia. Furthermore, at the end of the execution of its stages, an overview can be produced to demonstrate the evidences found, contributing with greater clarity in the applicability of probiotics as a therapeutic resource against dyslipidemia, which may point out gaps and stimulate further research in this area.

\section{Additional files}

Additional file 1: PRISMA-P (Preferred Reporting Items for Systematic review and Meta-Analysis Protocols) 2015 checklist. Recommended items to address in a systematic review protocol. (DOCX $41 \mathrm{~kb}$ )

Additional file 2: PubMed search. Combination of keywords and entry terms for search in the PUBMED (DOCX $14 \mathrm{~kb}$ )

Additional file 3: AMSTAR 2. Tool that will be used for critically appraising the systematic reviews included (PDF $250 \mathrm{~kb}$ )

\section{Abbreviations}

AMI: Acute myocardial infraction; AMSTAR: A Measurement Tool to Assess Systematic Reviews; CA: Covered area; CCA: Corrected covered area; CRF: Cardiovascular risk factors; HDL-c: High-density lipoprotein cholesterol; JBI: Joanna Briggs Institute; LDL-c: Low-density lipoprotein cholesterol; MA: Meta-analyze; MeSH: Medical Subject Headings; PICO: Population, Intervention, Comparison, Outcome(s); PRISMA: Preferential Reporting Items for Systematic Reviews and Meta-analyses; PRISMA-P: Preferred Reporting Items for Systematic Review and Meta-analysis-Protocol; RCT: Randomized controlled trials; SR: Systematic review; TIAB: Title/Abstract; WHO: World Health Organization

\section{Authors' contributions}

All authors, PMF, SMM, KAV, AVN, LRC, and PSC, were involved in the intellectual production and protocol design. The steps of searching, extracting, and synthesizing data proposed in the protocol will be developed by the authors PMF, KAV, and SMM under the coordination of LRC, AVN, and PSC. At the end of the development of the steps described in the protocol, the authors PMF, SMM, KAV, LRC, AVN, and PSC will carry out the analysis, interpretation, and discussion of the results for the production of an overview. The reviewers PMF and PSC were responsible for writing the protocol. All authors read and approved the final manuscript.

\section{Authors' information}

PMF-MD, MS, PhD Student. Pediatric, in Pediatric Nephrology. SMM-MD, PhD. Professor and author of papers based on systematic reviews.

KAV-MS, PhD Student. Author of papers based on systematic reviews. LRC-MS, PhD. Professor, certified by the Joanna Briggs Institute on the Systematic Reviews methods.

AVN-MD, PhD. Associate Professor in Pediatric Nephrology. PSC-MD, PhD. Associate Professor, certified by the Joanna Briggs Institute on the Systematic Reviews methods. 


\section{Ethics approval and consent to participate}

Not applicable.

\section{Consent for publication}

Not applicable.

\section{Competing interests}

The authors declare that they have no competing interests.

\section{Publisher's Note}

Springer Nature remains neutral with regard to jurisdictional claims in published maps and institutional affiliations.

\section{Author details}

${ }^{1}$ Health Sciences Graduate Program, Medicine School, Universidade Federal de Goiás (UFG), Rua 235, s/n, Setor Leste Universitário, Goiânia, GO CEP 74605-050, Brazil. ${ }^{2}$ Department of Pediatrics, Hospital das Clinicas, UFG, Primeira Avenida, s/n, Setor Leste Universitário, Goiânia, GO CEP 74605-020, Brazil. ${ }^{3}$ Dentistry Graduate Program, UFG, Primeira Avenida, s/n, esquina com Praça Universitária, Setor Leste Universitário, Goiânia, GO CEP 74605-220, Brazil. ${ }^{4}$ Division of Pediatric Dentistry, Faculdade de Odontologia, UFG, Primeira Avenida, s/n, esquina com Praça Universitária, Setor Leste Universitário, Goiânia, GO CEP 74605-220, Brazil. ${ }^{5}$ Health Education Graduate Program, Medicine School, UFG, Rua 235, s/n, Setor Leste Universitário, Goiânia, GO CEP 74605-050, Brazil.

Received: 9 March 2018 Accepted: 25 September 2018

Published online: 17 October 2018

\section{References}

1. WHO. World Health Organization. Cardiovascular disease. World heart day 2017. 2017. www.who.int/cardiovascular_diseases/world-heart-day-2017/en/. Accessed 16 Oct 2017

2. Nayor M, Vasan RS. Recent update to the us cholesterol treatment. Guidelines a comparison with international guidelines. Circulation. 2016;133: 1795-806. https://doi.org/10.1161/CIRCULATIONAHA.116.021407.

3. Ezzati M, Lopez AD, Rodgers A, Hoorn SV, Murray CJL, the Comparative Risk Assessment Collaborating Group. Selected major risk factors and global and regional burden of disease. Lancet. 2002;360(9343):1347-60. https://doi.org/ 10.1016/S0140-6736(02)11403-6.

4. Al-Hamad D, Raman V. Metabolic syndrome in children and adolescents Transl Pediatr. 2017;6(4):397-407. https://doi.org/10.21037/tp.2017.10.02.

5. Bowry ADK, Lewey J, Dugani SB, Choudhry NK. The burden of cardiovascular disease in low and middle-income countries: epidemiology and management. Can J Cardiol. 2015;31(9):1151-9. https://doi.org/10.1016/ j.cjca.2015.06.028.

6. Bilen O, Kamal A, Virani SS. Lipoprotein abnormalities in south Asians and its association with cardiovascular disease: current state and future directions. World J Cardiol. 2016;8(3):247-57. https://doi.org/10.4330/wjc.v8.i3.247.

7. FAO/WHO. Food and agriculture Organization of the United Nations and World Health Organization. In: Guidelines for the evaluation of probiotics in food; 2002. http://www.who.int/foodsafety/fs_management/en/probiotic_ guidelines.pdf. Accessed 05 Mar 2016.

8. Kechagia M, Basoulis D, Konstantopoulou S, Dimitriadi D, Gyftopoulou K, Sharmoutsou N, Fakiri EM. Health benefits of probiotics: a review. ISRN Nutrition. 2013;2013:ID481651. https://doi.org/10.5402/2013/481651.

9. Reis A, Conceição LL, Rosa DD, Siqueira NP, Peluzio CG. Mechanisms responsible for the hypocholesterolaemic effect of regular consumption of probiotics. Nutr Res Rev. 2017;30(1):36-49. https://doi.org/10.1017/ S0954422416000226.

10. Jones ML, Martoni CJ, Prakash S. Cholesterol lowering and inhibition of sterol absorption by Lactobacillus reuteri NCIMIB 30242: a randomized controlled trial. Eur J Clin Nutr. 2012;66(11):1234-41. https://doi.org/10.1038/ejcn.2012.126.

11. Fuentes MC, Lajo T, Currión JM, Cuñé J. A randomized controlled trial a proprietary mixture of Lactobacillus plantarum strains for lowering cholesterol. Med J Nutrition Metab. 2016;9(2):125-35. https://doi.org/10. 3233/MNM-160065.

12. Huang Y, Wang X, Wang J, Wu F, Sui Y, Yang L, Wang Z. Lactobacillus plantarum strains as potential probiotic cultures with cholesterollowering activity. J Dairy Sci. 2013;96(5):2746-53. https://doi.org/10.3168/ jds.2012-6123.
13. Roy CIL, Štšepetova J, Sepp E, Songisepp E, Claus SP, Mikelsaar M. New insights into the impact of Lactobacillus population on host-bacteria metabolic interplay. Oncotarget. 2015;6(31):30545-56.

14. Rerksuppaphol S, Rerksuppaphol L. A randomized double-blind controlled trial of Lactobacillus acidophilus plus Bifidobacterium bifidum versus placebo in patients with hypercholesterolemia. J Clin Diagn Res. 2015;9(3):KC01-4. https://doi.org/10.7860/JCDR/2015/11867.5728.

15. DiRienzo DB. Effect of probiotics on biomarkers of cardiovascular disease: implications for heart-healthy diets. Nutr Rev. 2014;72(1):18-29. https://doi. org/10.1111/nure.12084.

16. Greany KA, Bonorden MJL, Hamilton-Reeves JM, McMullen MH, Wangen KE, Phipps WR. At al. Probiotic capsules do not lower plasma lipids in young women and men. Eur J Clin Nutr. 2008;62:232-7. https://doi.org/10.1038/sj.ejcn.1602719.

17. Hatakka K, Mutanen M, Holma R, Saxelin M, Korpela M. Lactobacillus rhamnosus LC705 together with Propionibacterium freudenreichii ssp shermanii JS administered in capsules is ineffective in lowering serum lipids. J Am Coll Nutr. 2008:27(4):441-7.

18. Lee $Y, B a Z$, Roberts RF, Rogers CJ, Fleming JA, Meng H, et al. Effects of Bifidobacterium animalis subsp. Lactis BB-12 on the lipid/lipoprotein profile and short chain fatty acids in healthy young adults: a randomized controlled trial. Nutr J. 2017;16(1):39. https://doi.org/10.1186/s12937-017-0261-6.

19. Kasińska MA, Drzewoski J. Effectiveness of probiotics in type 2 diabetes: a meta-analysis. Pol Arch Med Wewn. 2015;125(11):803-13.

20. Hu Y-m, Zhou F, Yuan Y, Xu Y-c. Efectos del suplemento de probióticos en pacientes con diabetes mellitus tipo 2: metaanálisis de ensayos aleatorizados. Clin Med. 2016;148(8):362-70.

21. Cho YA, Kim JK. Effect of probiotic on bood lipid concentrations: a metaanalysis of randomized controlled trials. Medicine. 2015;94(43):e1714. https:// doi.org/10.1097/MD.0000000000001714

22. Wu Y, Zhang G, Ren Y, Ruan Z. Effect of probiotic Lactobacillus on lipid profile: a systematic review and meta-analysis of randomized controlled, trials. PLoS One. 2017;12(6):e0178868. https://doi.org/10.1371/journal.pone.0178868.

23. Aromataris E, Fernandez R, Godfrey C, Holly C, Khalil H, Tungpunkom P. Umbrella reviews. In: Aromataris E, Munn Z (Editors). Joanna Briggs Institute Reviewer's Manual. Adelaide: The Joanna Briggs Institute, 2017. https:// reviewersmanual.joannabriggs.org/display/MANUAL/. Accessed 02 Oct 2017.

24. Shamseer L, Moher D, Clarke M, Ghersi D, Liberati A, Petticrew M, et al. Preferred reporting items for systematic review and meta-analysis protocols (PRISMA-P) 2015: elaboration and explanation. BMJ. 2015;349:g7647. https:// doi.org/10.1136/bmj.g7647.

25. Higgins JPT, Green S. Guide to the contents of a Cochrane protocol and review. In: Higgins JPT, Churchill R, Chandler J, Cumpston MS, editors. Cochrane Handbook for Systematic Reviews of Interventions version 5.2.0. Chichester: The Cochrane Collaboration 2011. http://training.cochrane.org/ handbook. Accessed 05 Oct 2017.

26. Chandler J, Higgins JPT, Deeks JJ, Davenport C, Clarke MJ. Chapter 1: Introduction. In: Higgins JPT, Churchill R,Chandler J, Cumpston MS (editors). Cochrane Handbook for Systematic Reviews of Interventions version 5.2.0 (updated June 2017). Chichester: Cochrane; 2017.

27. Agerholm-Larsen L, Bell ML, Grunward GK, Astrup A. The effect of a probiotic milk product on plasma cholesterol: a meta-analysis of short-term intervention studies. Eur J Clin Nutr. 2000;54(11):856-60.

28. Moher D, Liberati A, Tetzlaff J, Altman DG, the PRISMA Group. Preferred reporting items for systematic reviews and meta-analyses: The PRISMA Statement. BMJ. 2009;339:b2535. https://doi.org/10.1136/bmj.b2535.

29. Withing P, Savovic J, Higgins JPT, Caldwell DM, Reeves BC, Shea B, Davies P, Kleijnen J, Churchill R, the ROBIS group. ROBIS: a new tool to assess risk of bias in systematic reviews was developed. J Clin Epidemiol. 2016;69:225-34. https://doi.org/10.1016/j.jclinepi.2015.06.005.

30. Shea BJ, Reeves BC, Wells G, Thuku M, Hamel C, Moran J, Moher D, Tugwell P, Welch V, Kristjansson E, Henry DA. AMSTAR 2: a critical appraisal tool for systematic reviews that include randomised or non-randomised studies of healthcare interventions, or both. BMJ. 2017;358:j4008. https:/doi.org/10.1136/bmj.j4008.

31. Pieper D, Antoine SL, Mathes T, Neugebauer EA, Eikermann M. Systematic review finds overlapping reviews were not mentioned in every other overview. J Clin Epidemiol. 2014;67(4):368-75.

32. Jadad AR, Cook DJ, Browman GP. A guide to interpreting discordant systematic review. Can Med Assoc J. 1997;156(10):1411-6. 